\title{
Bessel Functions of Real Argument and Integer Order
}

\author{
David J. Sookne ${ }^{1}$ \\ Institute for Basic Standards, National Bureau of Standards, Washington, D.C. 20234
}

(June 4, 1973)

A computer program is described for calculating Bessel Functions $J_{n}(x)$ and $I_{n}(x)$, for $x$ real, and $n$ a nonnegative integer. The method used is that of backward recursion, with strict control of error,

Key words: Backward recursion; Bessel functions; difference equation; error bounds; Miller algorithms.

\section{Method}

Given a real number $x$ and a positive integer $N B$, BESLRI calculates either

$$
I_{n}(x), \quad n=0,1, \ldots, \mathrm{NB}-1
$$

or

$$
J_{n}(x), \quad n=0,1, \ldots, \mathrm{NB}-1
$$

using double-precision arithmetic. The method, which is described in [1], ${ }^{2}$ is based on algorithms of Olver [2] and Miller [3], applied to the difference equation

$$
y_{n-1}=\frac{2 n}{x} y_{n}-\mathrm{SIGN} \cdot y_{n+1}
$$

where SIGN is +1 for $J$ 's, -1 for $I$ 's.

The program sets MAGX $=[|x|]$, the integer part of $|x|, p_{\mathrm{MAGX}}=0, p_{\mathrm{MAGX}+1}=1$, and then successively calculates

$$
p_{n+1}=\frac{2 n}{|x|} p_{n}-\mathrm{SIGN} \cdot p_{n-1} \quad n=\mathrm{MAGX}+1, \mathrm{MAGX}+2, \ldots .
$$

The sequence is strictly increasing. The program takes $N$ to be the least $n$ such that $p_{n}$ exceeds a number TEST defined in sections 2 and 3. It then sets $y_{N}^{(N)}=0, y_{N-1}^{(N)}=1 / p_{N}$, and recurs backward using (1). The computed sequence $y_{0}^{(N)}, y_{1}^{(N)}, \ldots$ is the recessive solution of (1) which satisfies the boundary condition $y_{\mathrm{MAGX}}=1$. From this solution, the $I$ 's and $J$ 's are found by normalizing

$$
\begin{array}{ll}
J_{n}(x)=y_{n}^{(N)} / \mu & n=0,1, \ldots, \mathrm{NB}-1 \\
I_{n}(x)=y_{n}^{(N)} / \mu & n=0,1, \ldots, \mathrm{NB}-1
\end{array}
$$

AMS Subject Classification: $68 \mathrm{~A} 10$.

1 Present address: W.U.J.S. Institute. Arad, Israel.

${ }^{2}$ Figures in brackets indicate the literature references at the end of this paper. 
where

$$
\begin{aligned}
& \mu=y_{0}^{(N)}+2 \sum_{k=1}^{[N / 2]} y_{2 k}^{(N)} \quad \text { for } J \text { 's } \\
& \mu=\left(y_{0}^{(N)}+2 \sum_{k=1}^{[N / 2]} y_{2 k}^{(N)}\right) / \cosh x \quad \text { for I's. }
\end{aligned}
$$

\section{Error Bounds for the $y_{n}$}

For $n>$ MAGX, the truncation error in $y_{n}^{(N)}$ is

$$
T_{n}^{(N)}=y_{n}-y_{n}^{(N)}=p_{n} \sum_{r=N}^{\infty} \frac{1}{p_{r} p_{r+1}}
$$

see [2], eqs (5.01) and (5.02). This error is bounded by using Lemma 2 of [1], which states that for $s \geqslant r, \rho_{s}=p_{s+1} / p_{s} \geqslant \min \left(p_{r+1} / p_{r},(r+1) /|x|+\sqrt{\left.(r+1)^{2} /|x|^{2}-1\right)}\right.$.

The program sets

$$
\mathrm{TEST}_{1} \geqslant \sqrt{2 \cdot 10^{\mathrm{NSIG}} p_{L} p_{L+1}}
$$

where $L=\max (\mathrm{MAGX}+1, \mathrm{NB}-1)$, and NSIG is the maximum number of significant decimal digits in a double-precision variable on the computer being used. Then $N^{\prime}$ is the least $n$ such that $p_{n}>$ TEST $_{1}$. For $J$ 's, $N$ is the least $n \geqslant N$ ' such that

$$
p_{n}>\operatorname{TEST}=\sqrt{\frac{\rho_{N^{\prime}}}{\rho_{N^{\prime}}^{2}-1}} \cdot \text { TEST }_{1} .
$$

In consequence of (4) and (5), the relative truncation error $\left|T_{n}^{(N)} / y_{n}\right|$ is less than $\frac{1}{2} \cdot 10^{- \text {NSIG }}$ for all $n$ in the range MAGX $<n \leqslant L$; see [1, sec. 5]. For $I$ 's, $N$ is taken to be $N$ '. Here a glance at (1) shows $p_{n} / p_{n-1}>2$ for all $n>$ MAGX, so the proof given in [1, sec. 5] for $J$ 's may also be used for I's, with $\rho_{N}$ replaced by 2 throughout.

In eq (1) for $I$ 's, $\frac{2 n}{x} y_{n}^{(N)}$ and $-\mathrm{SIGN} \cdot y_{n+1}^{(N)}$ have the same sign since if $x>0$, then $y_{n}^{(N)}>0$ for all $n$, and if $x<0$, then consecutive $y_{n}^{(N)}$ 's have opposite signs. Thus relative errors $<\epsilon$ in $y_{n+1}^{(N)}$ and $y_{n}^{(N)}$ will produce a relative error $<\epsilon$ in $y_{n-1}^{(N)}$. Therefore, the relative truncation error in all $I_{n}(x)$, $0 \leqslant x<\mathrm{NB}$, is bounded by $\frac{1}{2} \cdot 10^{-\mathrm{NSICi}}$.

For $J_{n}(x), n \leqslant$ MAGX, the relative truncation error cannot be bounded, since as $n=$ MAGX, MAGX $-1, \ldots, 1$ in (1), the values $J_{n}(x)$ oscillate, and precision is lost owing to cancellation. In this range, $J_{n}(x)$ is accurate to about $D$ decimal places, where $D$ is the number of decimals in $J_{\mathrm{MACX}+1}(x)$ which corresponds to NSIG significant figures in the same quantity [1, sec. 5$]$.

\section{Normalization and Error Bounds for $\mu$}

The eqs (3) were chosen to keep cancellation under control. First,

$$
\left|J_{0}(x)\right| \leqslant 1,\left|J_{n}(x)\right| \leqslant \frac{1}{\sqrt{2}}, \text { for } n \geqslant 1
$$


$[4,2.5]$, so each term of the sum (3) for $J$ 's is less than $\sqrt{2}$ times the whole sum. Since (6) is a rather weak bound, cancellation is even less than this would indicate.

For I's, cancellation is avoided altogether, since all terms in the sum (3) for I's have the same sign, as shown in section 2.

Besides bounding the truncation error of the algorithm, the program provides an estimated bound for the truncation error of the normalization sum, defined by eqs (3). For $J$ 's, this error is

$$
S^{(N)}=y_{0}-y_{0}^{(N)}+2 \sum_{k=1}^{\infty}\left(y_{2 k}-y_{2 k}^{(N)}\right) .
$$

For $2 k \leqslant$ MAGX, a bound for the error term $y_{2 k}^{(N)}-y_{2 k}$ is unavailable. For MAGX $<2 k<N$, $\left|y_{2 k}^{(N)}-y_{2 k}\right|<p_{2 k} \rho_{N} /\left(p_{N}^{2}-1\right)$ [1, sec. 5]. To avoid storing all the $p_{2 k}$, the program allows only for terms for which $2 k \geqslant N$. Here $y_{2 k}^{(N)}=0$, and $y_{2 k}=p_{2 k} \sum_{r=2 k}^{N} 1 /\left(p_{r} p_{r+1}\right)$. Therefore

$$
\begin{aligned}
& \left|y_{2 k}^{(N)}-y_{2 k}\right|=\left|\frac{1}{p_{2 k+1}}\left\{1+\frac{p_{2 k}}{p_{2 k+2}}+\frac{p_{2 k} p_{2 k+1}}{p_{2 k+2} p_{2 k+3}}+\ldots\right\}\right| \\
& \quad \leqslant \frac{1}{p_{2 k+1}}\left\{1+\frac{1}{\rho_{2 k}^{2}}+\frac{1}{\rho_{2 k}^{4}}+. .\right\}=\frac{\rho_{N^{\prime}}^{2}}{\left(\rho_{N^{\prime}}^{2}-1\right) p_{2 k+1}}
\end{aligned}
$$

compare section 2 above. Now let

$$
R^{(N)}=\sum_{k=\left[\frac{N+1}{2}\right]}^{\infty}\left|y_{2 k}\right| .
$$

Then

$$
\begin{gathered}
R^{(N) \leqslant \frac{\rho_{N^{\prime}}^{2}}{\rho_{N^{\prime}}^{2}-1} \sum_{k=\left[\frac{N+1}{2}\right]}^{\infty} \frac{1}{p_{2 k+1}}} \leqslant \frac{\rho_{N^{\prime}}^{2}}{\left(\rho_{N^{\prime}}^{2}-1\right) p_{N+1}}\left\{1+\frac{1}{\rho_{N^{\prime}}^{2}}+\frac{1}{\rho_{N^{\prime}}^{4}}+\ldots\right\} \\
\leqslant \frac{\rho_{N^{\prime}}^{3}}{\left(\rho_{N^{\prime}}^{2}-1\right)^{2} p_{N}} .
\end{gathered}
$$

For $J$ 's the program sets $\operatorname{TEST}_{1} \geqslant 2 \cdot 10^{\mathrm{NSIG}}$. The normalization factor $\mu$ is $1 / J_{\mathrm{MAGX}}(x)[1$, sec. 5$]$, so

$$
\left|\frac{R^{(N)}}{\mu}\right|=\left|J_{\mathrm{MAGiX}}(x)\right| R^{(N)} \leqslant \frac{\left|J_{\mathrm{MAGX}}(x)\right| \rho_{N^{\prime}}^{3}}{\left(\rho_{N^{\prime}}^{2}-1\right)^{2}} \cdot \frac{1}{2} \cdot 10^{-\mathrm{NSIG}} .
$$

This is a rather weak upper bound, and the error $\left|\frac{S^{(N)}}{\mu}\right|$ turns out to be less than $\frac{1}{2} \cdot 10^{- \text {NSICi }}$.

For $I$ 's, $p_{n} / p_{n-1}>2$ so the analog of (7) is

$$
R^{(N)} \leqslant \frac{8}{9 p_{N} \cosh x}
$$


this is derived by substituting 2 for $\rho_{N^{\prime}}$ in (7), and introducing the factor $\cosh x$; see (3).

By setting TEST $_{1} \geqslant \frac{2 \cdot 10^{-N S I G}}{\exp (0.461 \cdot \text { MAGX })}$, the program insures that

$$
R^{(N)} \leqslant \frac{4 \exp (0.461 \cdot \mathrm{MAGX})}{9 \cdot 10^{-\mathrm{NSI}} \cdot \cosh x}
$$

Here, $\mu$ is $\frac{1}{I_{\mathrm{MAGX}}}(x)$, so the relative error $\left|R^{(N)} / \mu\right|$ is bounded by

$$
\left|\frac{R^{(N)}}{\mu}\right| \leqslant \frac{4 I_{\mathrm{MAGX}}(x) \exp (0.461 \cdot \mathrm{MAGX})}{9 \cosh x \cdot 10^{-\mathrm{NSIG}}}
$$

Now Kapteyn's inequality $[4,8.7]$ states that

$$
\left|J_{n}(n z)\right| \leqslant\left|\frac{z \exp \sqrt{1-z^{2}}}{1+\sqrt{1-z^{2}}}\right|^{n}
$$

Let $z=\frac{|x|}{\mathrm{MAGX}}$, so for large $x, z=1$, approximately.

Then

$$
\begin{aligned}
\left|I_{\mathrm{MAGX}}(x)\right|=\left|J_{\mathrm{MAGX}}(i x)\right| & =\left|J_{\mathrm{MAGX}}(\operatorname{MAGX} \cdot i z)\right| \\
& \leqslant\left|\frac{z \exp \sqrt{1+z^{2}}}{1+\sqrt{1+z^{2}}}\right|^{\mathrm{MAGX}} .
\end{aligned}
$$

For large $x$, the approximate bound for $I_{\mathrm{MAGX}}(x)$ is

$$
\left(\frac{\exp \sqrt{2}}{1+\sqrt{2}}\right)^{\text {MAGX }}<\exp (0.533 \cdot \text { MAGX) }
$$

Substituting this in (8), we obtain

$$
\begin{aligned}
\left|\frac{R^{(N)}}{\mu}\right| & <\frac{4 \cdot \exp (0.533 \mathrm{MAGX}) \cdot \exp (0.461 \cdot \mathrm{MAGX})}{9 \cosh x \cdot 10^{-\mathrm{NSIG}}} \\
& <\frac{4}{9} \cdot 10^{-\mathrm{NSIG}}<\frac{1}{2} \cdot 10^{-\mathrm{NSIG}} .
\end{aligned}
$$

Besides this approximate upper bound, the strict bounds

$$
\left|\frac{R^{(N)}}{\mu}\right|<7.6 \cdot 10^{-\mathrm{NSIG}} \quad|x| \geqslant 1
$$

and

$$
\left|\frac{R^{(N)}}{\mu}\right|<0.565 \cdot 10^{-\mathrm{NSIG}} \quad|x|<1
$$

may be derived by using Kapteyn's inequality to obtain a strict bound for $\left|I_{\mathrm{MAGX}+1}(x)\right|$. 


\section{Appendix: Algorithm BESLRI}

1. ELT BESLRI, 1.730222 , 58730

000001 000002 000003 000004 000005 000006 000007 $00000 B$ 000009

000010

000010

000011

000013

00001

000015

000016

0011017

000018

000019

000020

00002

000022

000022

000023

000024

000025

00002

000027

00002

000029

000030

00003

000032

000033

0000.35

000036

000037

000038

000039

000040

000041

000042

000043

000044

000045

000145

000046

000047

000043

000049

000050

000051

000052

000053

000054
SUBROUTINE BESLRI ( $X$, NB, IZE, B,NCALC)

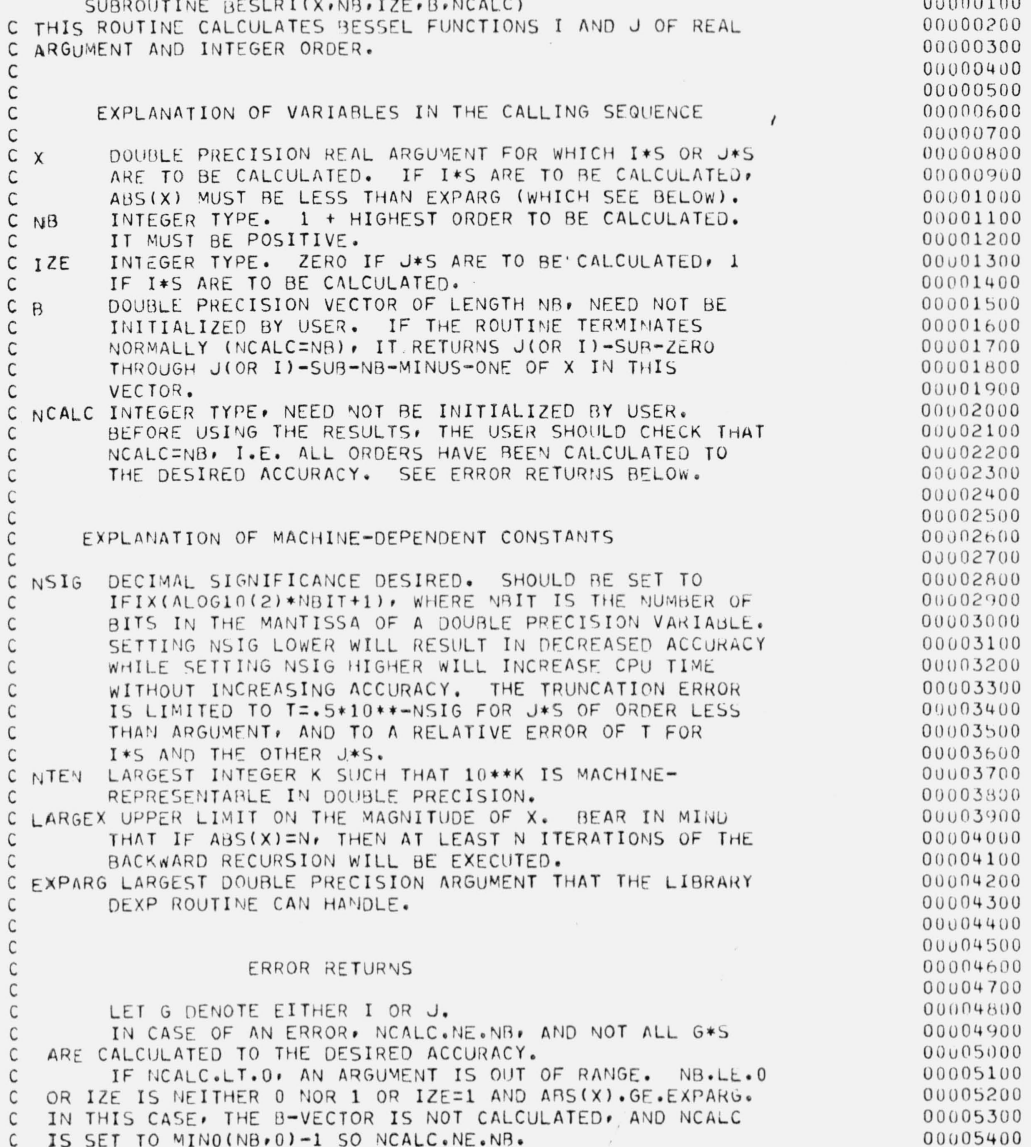


DOUISLE PRECISIOH

$1 X_{1}$, ,, TEST, TEMPA, TEMPB, TEMPC, EXPARG, SIGN, SUM, TOVER,

2 PLAST, POLD, PSAVE, PSAVEL

DIMENSION B(NB)

OATA NSIG,NIFN,LARGEX,EXPARG/19,307,100000,7.02/

TEMPA $=01935(X)$

"MAGX $=I F I X(\operatorname{SIN} L L(T E M P A))$

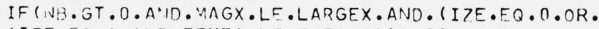

1(IZE.EO. 1.ANT. TEMPA.LF. EXPARG))) GO TO 1

C ERRDR RETURN - $x, N S, O R$ IZE IS OUT OF RANGE NCALC $\left.=\operatorname{MINO}(N)^{2}, 0\right)-1$ RETURN

1 SIGN=DBLE (FLOAT (1-2*IZE)) NCALC $=N B 3$

C USE 2-TERM ASCENDING SERIFS FOR SMALL $X$ IF (IFUPA**4

C INITIALIZE THE CALCULATION OF P*S

$$
\text { NAMX }=\text { NB-MAGX }
$$

$N=M A G X+1$

$N=V A G X+1$
$P L A S T=1 . P O$

$P L A S T=1.00$
$P=D J L E(F L O A T(2 * N)) / T F M P A$

C CALCULATE GLNERAL SIGNIFICANCE TEST

TEST $=2 \cdot 00 * 1 \cdot 01 * * N S I G$

IF (IZE. EU.1. AND.2*MAGX.GT.5*NSIG) TEST=DSQRT(TEST*P)

IF (IZE.EQ.1 - AND. 2*MAGX.LE. S*NSI IG) TEST TEST/1.585**MAGX $y=0$

IF (NFI $4 X \cdot L T \cdot 3)$ GO TO 4

C CALCULATE P*S UNTIL N=NH-1. CHECK FOR POSSIBLE OVERFLOW. TOVER $=1 \cdot 01 * *(N T E N-N S I G)$

NSTART $=M A G X+$ ?

NENU = N $B-1$

DO 3 NISNSTART, NEND

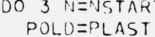

$P L A S T=P$

$P=D R L E(F L O A T(2 * N)) * P L A S T / T E M P A-S I G N * P O L D$

IF (P-TOVER) $3,3,5$

3 CONTINUE

$N=N E N D$

C CALCULATE SPECIAL SIGNIFICANCE TEST FOR NRMX.GT.?.

TEST = DMAX1 (TEST,DSQRT (PLAST*1.D1**NSIG)*DSQRT (2.D0*P))

C CALCULATE P*S UNTIL SIGNIFICANCE TEST PASSES

4 in $=N+1$

POLD $=P L A S T$

$P L A S T=P$

$P=D B L E(F L O A T(2 * N)) * P L A S T / T E M P A-S I G N * P O L D$

IF (P.LT.TEST) GO TO 4

IF (I ZE.E.3.1.OR.M.EQ.1) GO TO 12

C FOR J*S, A STRONG VARIANT OF THE TEST IS NECESSARY. 
TEMPE $=P / P L A S T$

TEMPC =DBLE (FLOAT $(N+1)) / T E M P A$

IF (TEMPB+1.DN/TEMPB.GT.2.DO*TEMPC) TEMPR=TEMPC+DSQR T 1 (TEMPC**2-1. DO)

TEST $=$ TEST $/ D S Q R T(T E M P B-1 \cdot D 0 / T E M P B)$

IF (P-TEST) $4,12,12$

C TO AVOIU OVERFLOW. DIVIDE P*S BY TOVER. CALCULATE P*S

TIL TOVET(P).GT

5 TOVER $=1 \cdot$ D 1**NTEN

PDPITOVER

PLAST $=P L A S T / T O V E R$

PSAVE=P

PSAVEL $=P L A S T$

NSTART $=N+1$

$6 \mathrm{~N}=\mathrm{N}+1$

POLDEPLAST

PLAST $=$ P

$P=D(3 L E(F L O A T(2 * N)) * P L A S T / T E M P A-S I G N * P O L D$

IF(P.LE.1.DO) GO TO G

TEMPG $=D R L E(F L O A T(2 * N)) / T E M P A$

IF (IZE.EQ.1) GO TO 8

IF $(1 Z E \cdot E Q \cdot 1)$ GO TO

TEMPC $=.5$ TEMPR $=P L A S T / P O M P B$

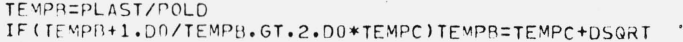
1 (TE:YPC **2-1.00)

C CALCULATE BACKWARD TEST, AND FIND NCALC, THE HIGHEST IN C SUCH THAT THE TEST IS PASSED.

8 TEST $=.500 * P O L D * P L A S T *(1.00-1.00 / T E M P B * 2) / 1.01 * * N S I G$ $P=P L A S T$ *TOVER

$N=N-1$

NENU) MINO (NB. N)

DO 9 NCALC $=$ NSTART, NEND

POLD $=$ PSAVEL

PSAVEL =PSAVE

PSAVE DDBLE (FLOAT (2*N)) *PSAVEL/TEMPA-SI GN*POLD

IF (PSAVE*PSAVEL-TEST) $9,9,10$

9 CONTINIJE

NCALC $=N E N D+1$

C THE SUM $3(1)+28(3)+2 B(5) \ldots$ IS USED TO NORMALIZE. M. THE

C THEFFICIFNT OF B(N), IS INITIALIZED TO $12 \mathrm{~N}=\mathrm{N}+1$

$N=N+1$
$M=2 * N-4 *(N / 2)$

C INITIALIZE THE BACKWARD RECURSION AND THE NORMALIZATION

c SUM

TEMP:3 $=0 \cdot 0$

TEMPA $=1 \cdot D 0 / P$

$S U M=D H L E(F L O A T(M)) * T E M P A$

NE: ND $=N-N-N / 3$

IF ( INFIOIO) $17,15,13$

C RECUIR BACKWARD VIA DIFFERENCE EQUATION, CALCULATING (BUT

C NOT STORING) B(N), UNTIL N=NB.

$130014 \mathrm{~L}=1$, NEND

$N=N-1$

$T E M P C=T E M P$

$T E$ MPB $=$ TEMPA

00011500

00011000

00011700

0011800

00011900

00012000

00012100

00012200

00012300
00012400

00012500

00012600

00012700

00012800

00012900

00013000

00013100

00013200

00013300

00013400

00013500

00013600

00013700

00013800

00013900

00014000

00014100

00014200

00014300

00014400

00014500

00014600

00014700

000148300

00014900

00015000

00015160

00015200

00015300

00015400

00015500

000156,00

00015700

00015800

00015900

00016000

00016100

$001) 16200$

00016300

00016300

00016500

00016600

00016700

00016800

00016900

00017000 


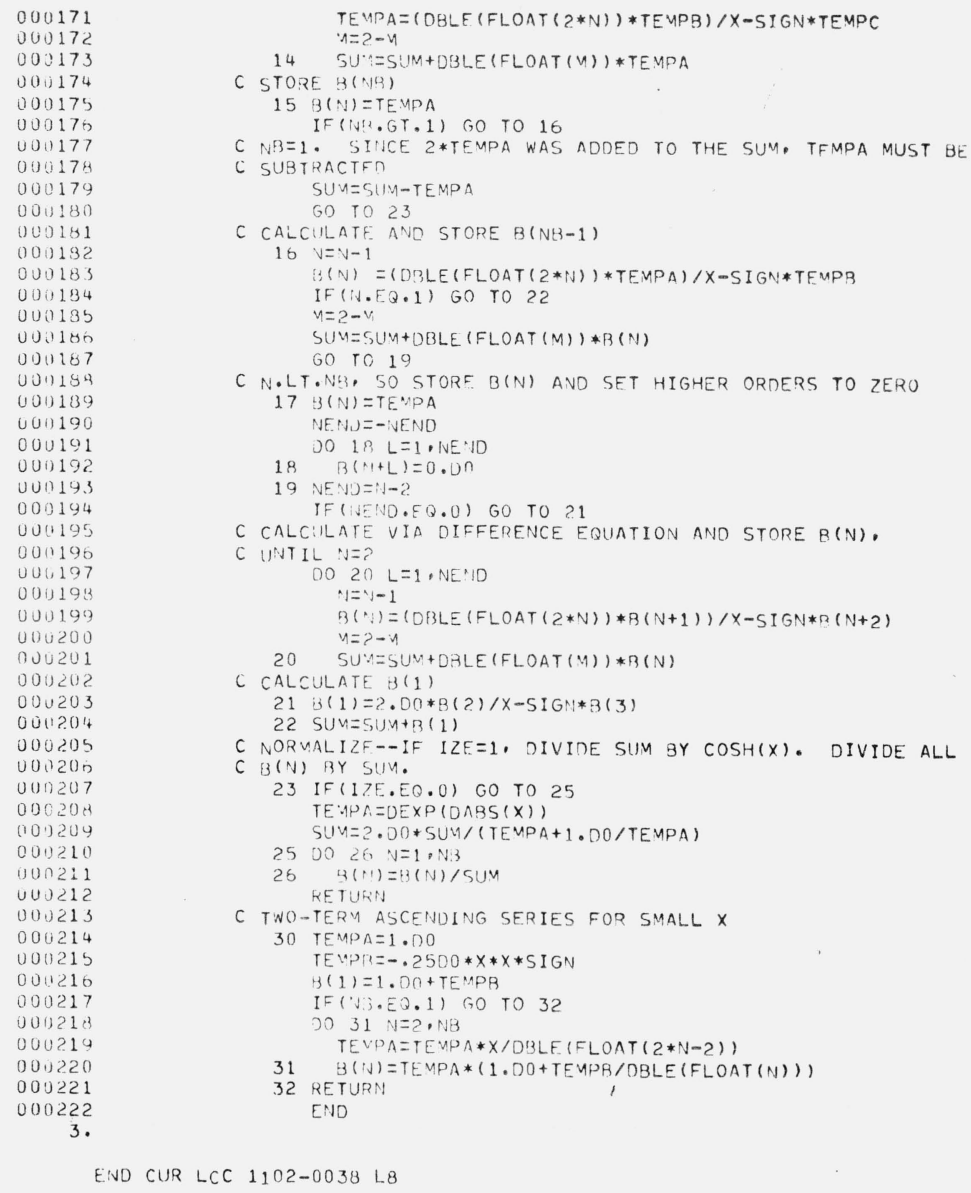

\section{References}

[1] Olver, F. W. J., and Sookne, D. J., A note on backward recurrence algorithms, Mathematics of Computation, Vol. 26, No. 120 (Oct. 1972), pp. 941-947.

[2] Olver, F. W. J., Numerical solution of second-order linear difference equations, J. Res. Nat. Bur. Stand. (U.S.), 71B, (Math. and Math. Phys.), Nos 2\&3, 111-129 (Apr.-Sept. 1967).

[3] British Assoc. for the Advancement of Science, Bessel Functions-Part II, Mathematical Tables, Vol. 10 (Cambridge University Press, Cambridge, 1952).

[4] Watson, G. N., A Treatise on the Theory of Bessel Functions (Second Edition, Cambridge University Press, Cambridge, 1944).

(Paper 77B3\&4-387) 